\title{
Does alpha-fetoprotein contribute to the mortality and morbidity of human hepatocellular carcinoma? A commentary
}

\author{
Gerald J Mizejewski \\ Wadsworth Center, \\ New York State Department \\ of Health, Albany, NY, USA
}

\author{
This article was published in the following Dove Press journal: \\ Journal of Hepatocellular Carcinoma \\ 21 September 2016 \\ Number of times this article has been viewed
}

Correspondence: Gerald J Mizejewski Wadsworth Center, New York State Department of Health, PO Box 509. Empire State Plaza, Albany, NY 12201-0509, USA

Tel + I 5184865900

$\mathrm{Fax}+\mathrm{I} 5 \mathrm{I} 4025002$ Email gerald.mizejewski@health.ny.gov

\begin{abstract}
The fifth most common cancer worldwide is hepatocellular carcinoma (HCC), while being the third leading cause of global cancer-related deaths. Although HCC incidence is less frequent in North America, it is a common malignancy in Asia and Africa associated with a high rate of mortality and morbidity due to ineffective therapies against cancer growth, invasion, and metastasis. It is well established that serum alpha-fetoprotein (AFP) is the "gold standard" biomarker for liver cancer; however, less known are the biological activities of AFP regarding carcinogenesis, growth, proliferation, and metastasis. Clinicians are well aware that increasing AFP serum levels parallel disease progression of HCC patients, but many are less knowledgeable in the lethal growth-promoting properties of AFP as an autocrine stimulator of hepatoma cell proliferation. This commentary addresses the mortality and morbidity concerning AFP in the genesis, growth, progression, and spread of HCC and emphasizes the perilous consequences of AFP-supported growth in human liver cancer even after liver resection and transplantation. Thus, AFP is not just a biomarker for HCC but also an ardent promoter of liver cancer growth and progression.
\end{abstract}

Keywords: growth, metastasis, carcinogenesis, proliferation, cancer

\section{Commentary}

Alpha-fetoprotein (AFP) serum concentrations in adults, similar to fetal AFP, have long served as a biomarker for distress, dysfunction, and presence of abnormal growth and anatomical malformations. ${ }^{1}$ During pregnancy, AFP serum levels are indicators of fetal defects and chromosomal abnormalities, while in adults, elevated AFP levels designate the presence of liver cirrhosis, viral hepatitis, and hepatocellular carcinoma (HCC). ${ }^{2}$ Although less familiar in the clinic, a major physiological property of AFP is that of a growth factor that is advantageous for the fetus by promoting growth and cell proliferation. However, the same beneficial trait of AFP in adults is extremely deleterious and lethal when associated with cellular transformation, growth, proliferation, and metastasis of liver cancer. The objective of the present commentary is to communicate a call of alert to the biomedical community of just how hazardous the AFP growth factor effect can be to the well-being of liver cancer patients. Although most clinicians are well informed of the correlation of elevated AFP levels with increasing severity of disease (ie, cancer), recent discoveries in the AFP-to-cancer biological relationships have greatly increased our knowledge concerning potential health threats to HCC patients. These newly revealed AFP reports in vitro, in vivo, and in patients 
have encompassed carcinogenesis, growth, proliferation, invasion, and metastasis of human liver cancer.

\section{Hepatocarcinogenesis}

Recent developments in the last decade have provided increased insight into the physiological role of extracellular (secreted) and intracytoplasmic (cell bound) AFP forms involved in all aspects of the genesis and progression of HCC. ${ }^{3}$ At the onset of hepatocarcinogenesis, AFP is regulated early in the process of cell transformation of liver parenchymal cells into hepatoma cells; moreover, AFP contributes to various malignant cell behaviors. ${ }^{4}$ In hepatitis $\mathrm{B}(\mathrm{HB})$ virusinfected liver cells, AFP is known to mediate HBx protein activity during the induction of liver carcinogenesis. ${ }^{5,6}$ The AFP receptor signal pathway has also been reported to be a contributor to $\mathrm{HBx}$-driven hepatocarcinogenesis. ${ }^{7}$ AFP was further found capable of blocking the expression of the retinoic acid receptor whose presence can interfere with Fn14 gene induction to promote apoptosis, angiogenesis, and proliferation of endothelial cells during the process of hepatocarcinogenesis. ${ }^{8}$ The Fn14 gene products are also known to be stimulators of hepatoma growth signal transduction pathways. Finally, AFP was found to activate AKT (protein kinase B) acting on mTOR in liver cells transfected with the HBx protein, thereby promoting malignant cell transformation via activation of PI3K together with mTOR. ${ }^{9}$

\section{HCC growth and proliferation}

Regarding the growth of cancer cells, AFP has been well documented in promoting the growth and proliferation of HCC cells. Toward this end, AFP has been reported to upregulate in vitro the growth and progression of HCC cells by $120 \%-150 \% .^{10,11}$ In addition, AFP is known to influence growth via cell cycle progression by regulation of the G1- to S-phase transition stage. ${ }^{12}$ To further maintain cancer growth and progression, AFP promotes HCC-related angiogenesis of new blood vessels to supply nutrients to the tumor. ${ }^{13} \mathrm{AFP}$ is also able to inhibit apoptosis of HCC cells, thus increasing cancer cell population numbers. ${ }^{14}$ AFP has also been known to shield HCC cells from tumor necrosis factor-induced cell death $^{15}$ and to promote escape of tumor cells from lymphocyte cytotoxic cells via the caspase enzyme pathways. ${ }^{16}$ In this regard, AFP is capable of physically binding to caspase-3, but not caspase- 8 or $-9 .{ }^{17}$ In the enhancement of HCC cell proliferation, AFP can upregulate KRas, cyclic adenosine monophosphate, and protein kinase $\mathrm{A}$ and increase cytosolic $\mathrm{Ca}^{++}$levels. ${ }^{8,18}$ Thus, AFP cooperates with both growth factors and transcription factors to promote $\mathrm{HCC}$ cell growth. ${ }^{8}$ In the regulation of $\mathrm{PI} 3 \mathrm{~K} / \mathrm{AKT}$ activation, AFP is able to promote degradation of the p27 cell cycle inhibitor by means of ubiquitin ligases, thus advancing cell cycle progression. ${ }^{19}$ Finally, during the AFP interaction in retinoic acid receptor signaling pathways, the repression of GAAD153 (growth arrest and DNA damage-inducible protein-153) serves to enhance HCC growth. ${ }^{20}$

\section{HCC invasion and metastasis}

AFP has recently been reported to play a crucial role in HCC cell metastasis and invasion. ${ }^{21}$ In that report, high serum levels of AFP were positively correlated with HCC intrahepatic invasion and extrahepatic lung node and lung metastasis. The investigators found that AFP promoted the expression of four metastasis-related proteins, including keratin-19, epithelial cell adhesion molecule, matrix metalloproteinases 2 and 9, and CXC chemokine receptor-4. The latter chemokine receptor plays a major chemoattractant role by directing cancer cells to home into specific organs, that is, lymph nodes, bone marrow, and lungs. In further studies, AFP was reported to activate AKT (protein kinase B) and mTOR to induce expression of $\mathrm{CXC}$ chemokine receptor-4, which initiates metastasis. ${ }^{22}$ Overall, AFP governs the expression of several metastasis-related genes. Elevated serum AFP levels in HCC patients are further known to correlate with metastasis of HCC cells. ${ }^{23}$ These cancer patients' serum AFP levels were higher in HCC metastasis patients than in sera of patients with hepatic trauma and dysfunction. Furthermore, AFP was reported to promote distant metastasis of HCC cells implanted in mouse xenograft models. ${ }^{21}$ Thus, AFP harbors a function to aid in $\mathrm{HCC}$ cell migration and invasion by several means, one of which is to stimulate expression of metastasisrelated proteins. Finally, AFP messenger RNA expression was found in $60 \%$ of circulating tumor cells of HCC patients diagnosed with metastasis. ${ }^{24}$ It has become obvious that AFP is an active agent in HCC invasion and metastasis.

\section{HCC in liver resection and transplantation}

Liver resection in $\mathrm{HCC}$ patients is a potentially curative, low-cost medical procedure if performed soon after HCC diagnosis. In contrast, liver transplantation can include long waiting periods and higher surgical costs requiring patients who display preserved liver function. Although there are no established cutoff values for serum AFP levels following liver resection and transplantation, published reports indicate 
that the serum AFP clinical outcomes discussed earlier hold true to concept. Following liver resection, serum AFP levels $>400 \mathrm{ng} / \mathrm{mL}$ signify a higher risk of microvascular invasion in liver tissue, HCC recurrence, and poor patient survival. ${ }^{25}$ In cases of liver transplantation after primary liver resection, serum AFP levels $<200 \mathrm{ng} / \mathrm{mL}$ were associated with lower HCC recurrence and higher patient survival rates. ${ }^{26}$ As shown in this report, lower serum AFP levels favor increased patient survival times, while increased serum AFP levels reflect a detriment to patient health and well-being.

\section{Conclusion}

It is now evident that serum and cytoplasmic AFP are not just bystander agents but are active participants in all phases of transformation, growth, proliferation, and metastasis of the HCC malignant state. Thus, AFP is intrinsically involved in the overall process of cancer progression from carcinogenesis to metastasis. The earlier discussion demonstrates that AFP is a lethal compound involved in HCC cell apoptosis, shielding, lymphocyte escape, cell cycle progression, and signal transduction pathways. In many of the reports cited earlier, ${ }^{15-17}$ knockout of the AFP gene inhibited and repressed HCC tumor growth, while the transfection of the AFP gene into non-AFP hepatomas initiated HCC tumor genesis, growth, and progression. It is apparent that the absence of AFP from the tumor environment eliminates much of the tumor lethality and reduces HCC growth, while AFP presence adds to the lethality of tumor presence. Although AFP is known as the "gold standard" biomarker for HCC, it must be emphasized that AFP itself is a highly effective growth factor and not all clinicians may be aware of its immense potential for enhancing HCC growth. Indeed, it has recently been reported that AFP contains three epidermal growth factor motifs, one on each domain of the AFP polypeptide. ${ }^{27}$ Although AFP greatly contributes to fetal growth during pregnancy, full-length AFP can now be documented to play a lethal role in the growth and progression of HCC. In conclusion, it is of utmost importance that recombinant AFP should not be employed as a therapeutic agent to treat human disease (cancer, autoimmune disorders, etc). Since AFP is a "loose cannon" bristling with protein-to-protein interaction sites, it would be instead more prudent to use AFP-derived domain fragments and/or peptide segments for any type of therapy in humans. Hypothetically, the ultimate therapy for HCC should include 1) downregulation of the AFP gene, 2) use of microRNA and small interfering RNA against AFP, and/or 3) AFP vaccination against HCC.

\section{Disclosure}

The author reports no conflicts of interest in this work.

\section{References}

1. Mizejewski GJ. Biological roles of alpha-fetoprotein during pregnancy and perinatal development. Exp Biol Med. 2004;229(6):439-463.

2. Mizejewski GJ. Biological role of alpha-fetoprotein in cancer: prospects for anticancer therapy. Expert Rev Anticancer Ther. 2002;2(6):709-735.

3. Mizejewski GJ. Nonsecreted cytoplasmic alpha-fetoprotein: a newly discovered role in intracellular signaling and regulation. An update and commentary. Tumor Biol. 2015;36(12):9857-9864.

4. Hung TM, Hu RH, Ho CM, et al. Downregulation of alpha-fetoprotein expression by LHX4: a critical role in hepatocarcinogenesis. Carcinogenesis. 2011;32(12):1815-1823.

5. Arima T, Nakao K, Nakata K, et al. Transactivation of human alphafetoprotein gene by X-gene product of hepatitis B virus in human hepatoma cells. Int J Mol Med. 2002;9(4):397-400.

6. Zhang C, Chen X, Liu H, et al. Alpha fetoprotein mediates HBx induced carcinogenesis in the hepatocyte cytoplasm. Int $J$ Cancer. 2015;137(8):1818-1829.

7. Zhu M, Guo J, Li W, et al. HBx induced AFP receptor expressed to activate PI3K/AKT signal to promote expression of Src in liver cells and hepatoma cells. BMC Cancer. 2015;15:362-370.

8. Wang S, Jiang W, Chen X, et al. Alpha-fetoprotein acts as a novel signal molecule and mediates transcription of Fn14 in human hepatocellular carcinoma. $J$ Hepatol. 2012;57(2):322-329.

9. Zhu M, Guo J, Li W, et al. Hepatitis B virus X protein induces expression of alpha-fetoprotein and activates PI3K/mTOR signaling pathway in liver cells. Oncotarget. 2015;6(14):12196-12208.

10. Wang XW, Xie H. Alpha-fetoprotein enhances the proliferation of human hepatoma cells in vitro. Life Sci. 1999;64(1):17-23.

11. Li MS, Li PF, He SP, Du GG, Li G. The promoting molecular mechanism of alpha-fetoprotein on the growth of human hepatoma Bel7402 cell line. World J Gastroenterol. 2002;8(3):469-475.

12. Tang H, Tang XY, Liu M, Li X. Targeting alpha-fetoprotein represses the proliferation of hepatoma cells via regulation of the cell cycle. Clin Chim Acta. 2008;394(1-2):81-88.

13. Mitsuhashi N, Kobayashi S, Doki T, et al. Clinical significance of alphafetoprotein: involvement in proliferation, angiogenesis, and apoptosis of hepatocellular carcinoma. J Gastroenterol Hepatol. 2008;23(7 pt 2):e189-e197.

14. Laderoute MP, Pilarski LM. The inhibition of apoptosis by alpha-fetoprotein (AFP) and the role of AFP receptors in anti-cellular senescence. Anticancer Res. 1994;14(6B):2429-2438.

15. Li M, Zhou S, Liu X, Li P, McNutt MA, Li G. alpha-Fetoprotein shields hepatocellular carcinoma cells from apoptosis induced by tumor necrosis factor-related apoptosis-inducing ligand. Cancer Lett. 2007;249(2):227-234.

16. Li M, Liu X, Zhou S, Li P, Li G. Effects of alpha fetoprotein on escape of Bel 7402 cells from attack of lymphocytes. BMC Cancer. 2005;5:96-106.

17. $\mathrm{Li} \mathrm{M}, \mathrm{Li} \mathrm{H}, \mathrm{Li} \mathrm{C}$, et al. Alpha fetoprotein is a novel protein-binding partner for caspase- 3 and blocks the apoptotic signaling pathway in human hepatoma cells. Int J Cancer. 2009;124(12):2845-2854.

18. Wang XW, Xu B. Stimulation of tumor-cell growth by alpha-fetoprotein. Int J Cancer. 1998;75(4):596-599.

19. Li M, Li H, Li C, et al. Alpha-fetoprotein: a new member of intracellular signal molecules in regulation of the PI3K/AKT signaling in human hepatoma cell lines. Int J Cancer. 2011;128(3):524-532.

20. Li C, Wang S, Jiang W, et al. Impact of intracellular alpha fetoprotein on retinoic acid receptors-mediated expression of GADD153 in human hepatoma cell lines. Int J Cancer. 2012;130(4):754-764.

21. Lu Y, Zhu M, Li W, et al. Alpha fetoprotein plays a critical role in promoting metastasis of hepatocellular carcinoma cells. J Cell Mol Med. 2016;20(3):549-558. 
22. Zhu M, Guo J, Xia H, et al. Alpha-fetoprotein activates AKT/mTOR signaling to promote CXCR4 expression and migration of hepatoma cells. Oncoscience. 2015;2(1):59-70.

23. Ohba T, Yano T, Yoshida T, et al. Results of a surgical resection of pulmonary metastasis from hepatocellular carcinoma: prognostic impact of the preoperative serum alpha-fetoprotein level. Surg Today. 2012;42(6):526-531.

24. Jin J, Niu X, Zou L, et al. AFP mRNA level in enriched circulating tumor cells from hepatocellular carcinoma patient blood samples is a pivotal predictive marker for metastasis. Cancer Lett. 2016;378(1):33-37.
25. Schlichtemeier SM, Pang TC, Williams NE, et al. A pre-operative clinical model to predict microvascular invasion and long-term outcome after resection of hepatocellular cancer: the Australian experience. Eur J Surg Oncol. Epub 2016 Jun 11, pages 1-8.

26. Yong CC, Tsai MC, Lin CC, et al. Comparison of salvage living donor liver transplantation and local regional therapy for recurrent hepatocellular carcinoma. World J Surg. Epub 2016 May 18, pages 1-9.

27. Mizejewski GJ. The alpha-fetoprotein third domain receptor binding fragment: in search of scavenger and associated receptor targets. $J$ Drug Target. 2015;23(6):538-551.

\section{Publish your work in this journal}

The Journal of Hepatocellular Carcinoma is an international, peerreviewed, open access journal that offers a platform for the dissemination and study of clinical, translational and basic research findings in this rapidly developing field. Development in areas including, but not limited to, epidemiology, vaccination, hepatitis therapy, pathology and

Submit your manuscript here: https://www.dovepress.com/journal-of-hepatocellular-carcinoma-journa

molecular tumor classification and prognostication are all considered for publication. The manuscript management system is completely online and includes a very quick and fair peer-review system, which is all easy to use. Visit http://www.dovepress.com/testimonials.php to read real quotes from published authors. 